\title{
A Benchmarking Measurement Campaign to Support Ubiquitous Localization in GNSS Denied and Indoor Environments
}

\author{
Guenther Retscher, Allison Kealy, Vassilis Gikas, Jelena Gabela, Salil Goel, \\ Yan Li, Andrea Masiero, Charles K. Toth, Harris Perakis, \\ Wioleta Błaszczak-Bąk, Zoltan Koppanyi, and Dorota Grejner-Brzezinska
}

\begin{abstract}
Localization in GNSS-denied/challenged indoor/outdoor and transitional environments represents a challenging research problem. As part of the joint IAG/FIG Working Groups 4.1.1 and 5.5 on Multi-sensor Systems, a benchmarking measurement campaign was conducted at The Ohio State University. Initial experiments have demonstrated that Cooperative Localization (CL) is extremely useful for positioning and navigation of platforms navigating in swarms or networks. In the data acquisition campaign, multiple sensor platforms, including vehicles, bicyclists and pedestrians were equipped with combinations of GNSS, Ultra-wide Band (UWB), Wireless Fidelity (Wi-Fi), Raspberry Pi units, cameras, Light Detection and Ranging (LiDAR) and inertial sensors for CL. Pedestrians wore a specially designed helmet equipped with some of these sensors. An overview of the experimental configurations, test scenarios, characteristics and sensor specifications is given. It has been demonstrated that all involved sensor platforms in the different test scenarios have gained a significant increase in positioning accuracy by using ubiquitous user localization. For example,
\end{abstract}

G. Retscher $(\bowtie)$

Department of Geodesy and Geoinformation, TU Wien - Vienna

University of Technology, Vienna, Austria

e-mail: guenther.retscher@tuwien.ac.at

A. Kealy

Department of Geospatial Science, RMIT University, Melbourne, VIC, Australia

e-mail: allison.kealy@rmit.edu.au

V. Gikas · H. Perakis

School of Rural and Surveying Engineering, National Technical

University of Athens, Athens, Greece

e-mail: vgikas@central.ntua.gr; hperakis@central.ntua.gr

J. Gabela

Department of Electrical and Electronic Engineering, The University of Melbourne, Melbourne, VIC, Australia

e-mail: jgabela@student.unimelb.edu.au

S. Goel

Department of Civil Engineering, Indian Institute of Technology,

Kanpur, Uttar Pradesh, India

e-mail: sgoel@iitk.ac.in

Y. Li

SMART Infrastructure Facility, University of Wollongong,

Wollongong, NSW, Australia

e-mail: liyan@uow.edu.au

\author{
A. Masiero \\ Interdepartmental Research Center of Geomatics, University of \\ Padova, Padova, Italy \\ e-mail: masiero@dei.unipd.it \\ C. K. Toth \\ Department of Civil, Environmental and Geodetic Engineering, The \\ Ohio State University, Columbus, OH, USA \\ e-mail: toth.2@osu.edu \\ W. Błaszczak-Bak \\ Institute of Geodesy of the University of Warmia and Mazury, Olsztyn, \\ Poland \\ e-mail: wioleta.blaszczak@uwm.edu.pl \\ Z. Koppanyi \\ Leica Geosystems, Heerbrugg, Switzerland \\ e-mail: zoltan.koppanyi@gmail.com \\ D. Grejner-Brzezinska \\ College of Engineering, The Ohio State University, Columbus, OH, \\ USA \\ e-mail: grejner-brzezinska.1@osu.edu
}


in the indoor environment, success rates of approximately $97 \%$ were obtained using WiFi fingerprinting for correctly detecting the room-level location of the user. Using UWB, decimeter-level positioning accuracy is demonstrable achievable under certain conditions. The full sets of data is being made available to the wider research community through the WG on request.

\section{Keywords}

Cooperative localization (CL) - GNSS-denied environments - Indoor positioning · Positioning - Navigation and Timing (PNT) - Relative ranging - Ultra-wideband (UWB) · Wireless Fidelity (Wi-Fi)

\section{Introduction and Objectives}

In GNSS challenged environments, an augmentation with other emerging positioning technologies is an unremitting requirement. This requirement led to the development of multi-sensor systems and their integration using sensor fusion. Thus, for ubiquitous positioning solutions several technologies are researched and further developed. One strategy is to use so-called wireless signals-of-opportunity which were originally not intended for positioning, such as Wireless Fidelity (Wi-Fi). Moreover, designated technologies based on pre-deployed signal transmission infrastructure as well as technologies not based on signals are developed and enhanced in the research conducted by the IAG Sub-Commission 4.1 in the last years. In the first category fall systems using infrared or ultrasonic signals, Ultra-wide Band (UWB), ZigBee, Radio Frequency Identification (RFID), Bluetooth, Light Emitting Diodes (LED), Dedicated Short Range Communication (DSRC) or other radio frequency (RF) based systems. Vision/camera systems as well as inertial sensors, such as accelerometers, gyroscopes, magnetometers, employed for dead reckoning belong to the second category. Also, a typical application field is smartphone positioning which plays an important role in the interdisciplinary research conducted under the umbrella of the SC 4.1. Furthermore, the Sub-Commission lays an emphasis on multi-sensor cooperative systems which employ all aforementioned variety of sensors on different platforms for sharing their absolute and relative locations. Platforms include mobile vehicles, robots as well as pedestrians and most recently UAS (Unmanned Aerial Systems). Their land and airborne navigation applications range from transportation, personal mobility, industrial and indoor positioning applications and to a lesser extent environmental monitoring. Thus, the major key objective of the SC is to examine the potential and capabilities of low-cost sensors including GNSS systems and smartphone navigation sensors. Primary sensors of interest include inertial and wireless technologies as well as vision-based systems and laser scanning for improving the navigation performance. Furthermore, other objectives include to contribute in research that depends on big data handling, sensor synchronization, data fusion, real-time processing as well as to support standardization activities and to study and monitor the progress of new multi-sensor applications, as well as, to support and promote knowledge exchange and reporting on the development trends, possibilities and limitations of emerging positioning technologies. Thereby the development of new measurement integration algorithms based around innovative modeling techniques in other research domains, such as machine learning and genetic algorithms, spatial cognition etc., plays also an important role.

\section{$2 \quad$ PNT Application Requirements}

Figure 1 provides an overview about the PNT (Positioning, Navigation and Timing) user requirements listed in a 'fish plot'. These requirements can be categorized in four different classes which are positioning, cost, security and legal as well as interface requirements. Thereby the most relevant positioning requirements in our view are apart from positioning accuracy also integrity, availability and coverage, latency and continuity as well as sampling and update rate. The other three requirements, however, also must not to be ignored. Operational and maintenance costs, for instance, are very important too when designing a low-cost positioning system. The GNSS Market Report of the European Commission in 2017 (GNSS Market Report 2017) identified the key GNSS requirements and performance parameters. Here they are also applied for alternative positioning technologies and techniques, such as UWB and Wi-Fi, as they are valid for any other PNT applications not involving only GNSS but also other sensors and technologies which are additionally and independently used (Retscher et al. 2020b). Regarding availability the number of transmitters (UWB stationary 


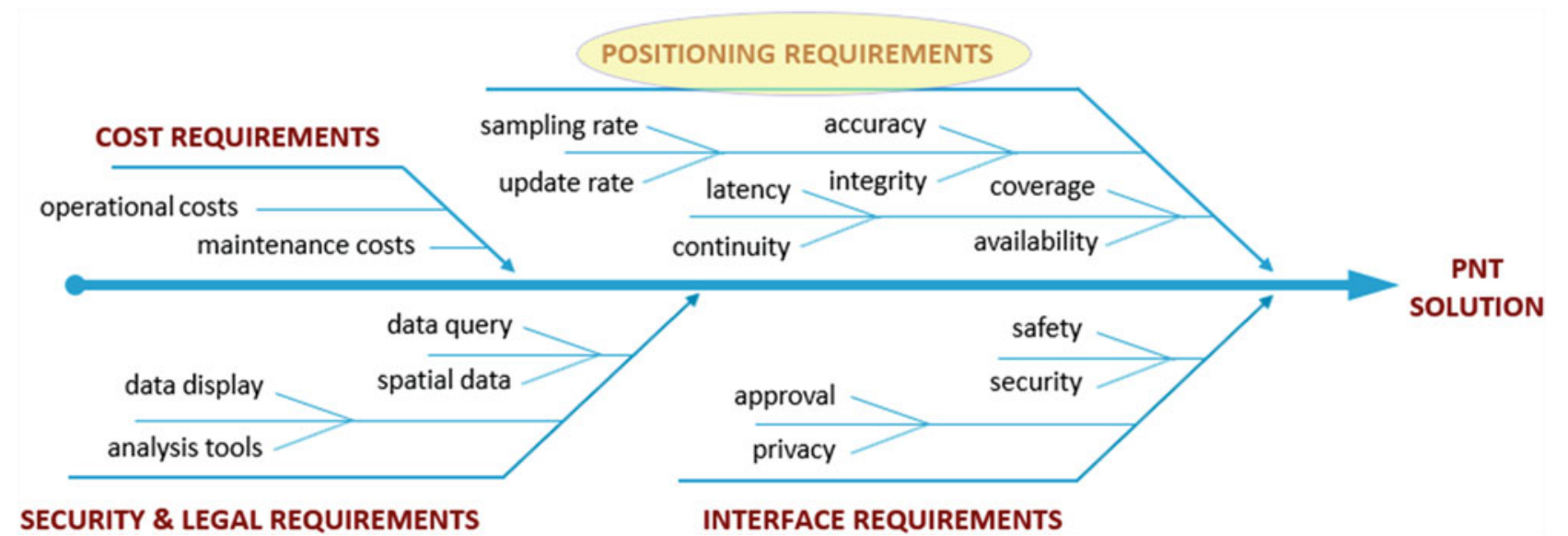

Fig. 1 Overview of PNT user requirements

transmitters or Wi-Fi Access Points) replaces the number of satellites. Especially integrity is often neglected and not paid full attention. It can be seen as a very important key parameter. The way that integrity is ensured and assessed, and the means of delivering integrity related information to the user are highly application dependent. Time-to-first-fix (TTFF) in the case of Wi-Fi positioning is highly correlated to the received signal strength (RSS) scan duration of a certain mobile device. This is especially important in kinematic positioning. As seen by the authors in Retscher and Leb (2019) the appearing scan durations can vary significantly for different smartphones which results in a different level of achievable positioning accuracy in dependence of the walking speed in the case of pedestrian navigation. For different users robustness may have a different meaning, such as the ability of the solution to respond following a server shadowing event. Here, robustness is defined as the ability of the solution to mitigate interference. Other requirements and performance parameters are power consumption, resiliency, connectivity, interoperability and traceability. Especially in the case of mobile devices power consumption is still very critical to provide a long-term solution possibility. Resiliency is the ability to prepare for and adapt to changing conditions, such as it is the case for Wi-Fi RSSI (Received Signal Strength Indicator) signal variations and fluctuations. To encounter for their influence new robust schemes are necessary and need to be developed.

\section{Field Campaign at The Ohio State University}

A benchmarking measurement campaign dealing with cooperative localization (CL) of different mobile sensor platforms navigating within a neighbourhood was conducted in October 2017 (Retscher et al. 2020a). Pedestrians as well as vehicle test were carried out. In the case of pedestrian CL, four pedestrians with a specially designed helmet equipped with GNSS, two UWB systems (i.e., from TimeDomain and Pozyx), Raspberry Pi, Wi-Fi and smartphone camera were moving around jointly, with the objective of achieving precise positioning in indoor environments, as well as providing a seamless position transition between indoor and outdoor environments. Relative range observations among pedestrians, camera observations, UWB range and Wi-Fi RSSI measurements were performed. All users transition from outdoor to indoor environments and thus, each pedestrian starts to lose GNSS signals successively. Once all pedestrians are indoors, GNSS observations are not available to any of them and therefore the users rely on relative UWB ranges, Wi-Fi measurements, and camera observations, for localizing all users cooperatively. For further details about the campaign the reader is referred to Kealy et al. (2019) and Retscher et al. (2020a).

\subsection{Indoor UWB Localization Results}

The UWB indoor experiment of the campaign aims at the investigation of the possibility of calibrating the UWB system in order to compensate for the effects of the static parts of the environment on UWB measurements, hence obtaining an improvement of the overall positioning accuracy (Retscher et al. 2020a). 14 Pozyx UWB anchors were fixed on the walls in the hallway and calibration and validation range measurement data sets were collected on 35 checkpoints along the corridor. 27 of these checkpoints were observed both during the calibration and validation data collection, whereas the remaining 8 were used only for validation. The users were moving in the test site in stopand-go mode, whereas for the calibration data collection only a few persons ( 3 to 4 , mostly involved in this experiment) 


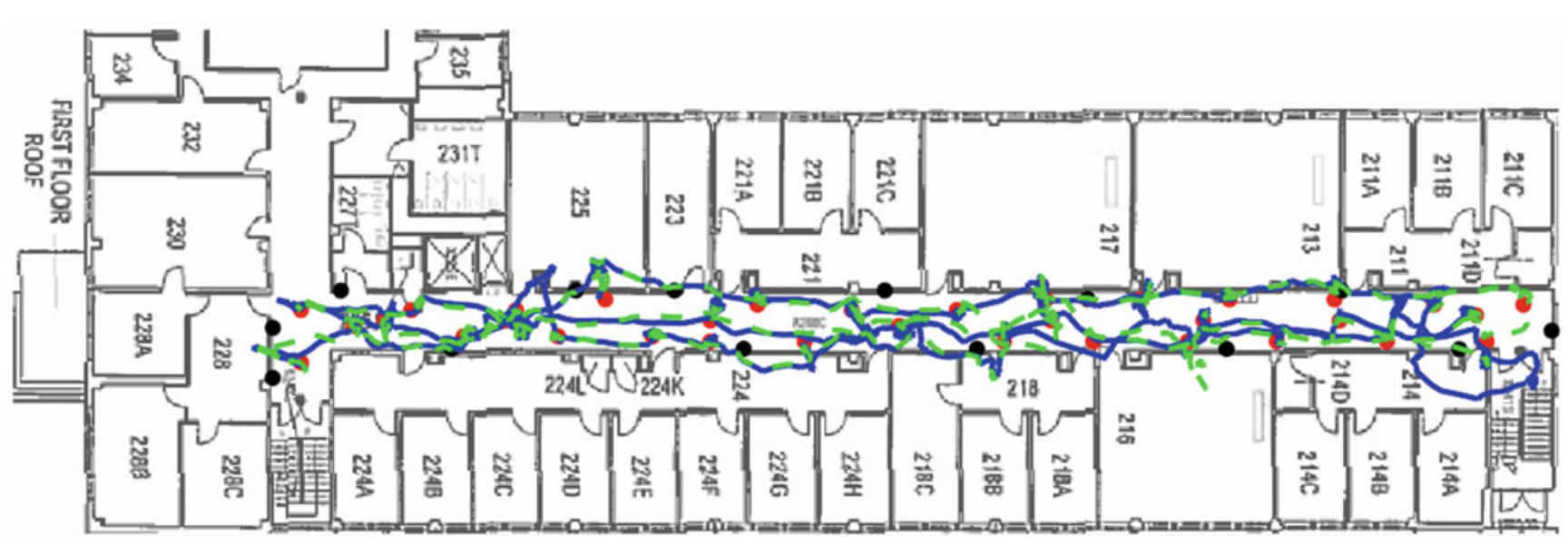

Fig. 2 Trajectory in the hallway estimated without calibration (solid blue line) and with the considered calibration model (green dashed line)

and in the validation up to 10 persons were moving around representing a more realistic and challenging scenario. For the calibration the median of the measured range errors was considered as an estimate of the measurement bias to be removed. Since the value of such a bias varies over all the area of interest, a calibration model with spatially varying additive bias was employed for each anchor by means of natural neighbour interpolation of the values computed on the 27 calibration points. Estimated trajectories obtained by a standard Extended Kalman filter are shown in Fig. 2 whereby either for the first trajectory no calibration (solid blue line) or for the second the aforementioned calibration model (dashed green line) are employed. A simple dynamic random walk model for the velocities of the device movement for integrating the ranges is assumed. Furthermore, the method took also advantage of a still-condition detection step similar as applied in Masiero et al. (2019). Figure 2 shows also anchor (black dots) and checkpoint (red dots) locations. As can be seen from the figure the improvement obtained with the proposed approach is quite significant and the considered approach can potentially be useful to reduce the effect of the systematic errors on the UWB measurements. Figure 3 presents the cumulative distribution function (CDF) of the 2D positioning errors for the results shown in Fig. 2. Distributions shown in Fig. 3 are obtained by taking into account of the 2D errors on the checkpoints shown as red dots in Fig. 2. Figure 3 confirms the improvement obtained with the considered calibration and tracking approach, e.g. the maximum 2D positioning error is almost $2 \mathrm{~m}$ smaller in the calibrated case with respect to the uncalibrated one.

\subsection{Wi-Fi Localization Results}

The aim of the Wi-Fi localization in the building was to achieve at least room-level or region-level granularity. For

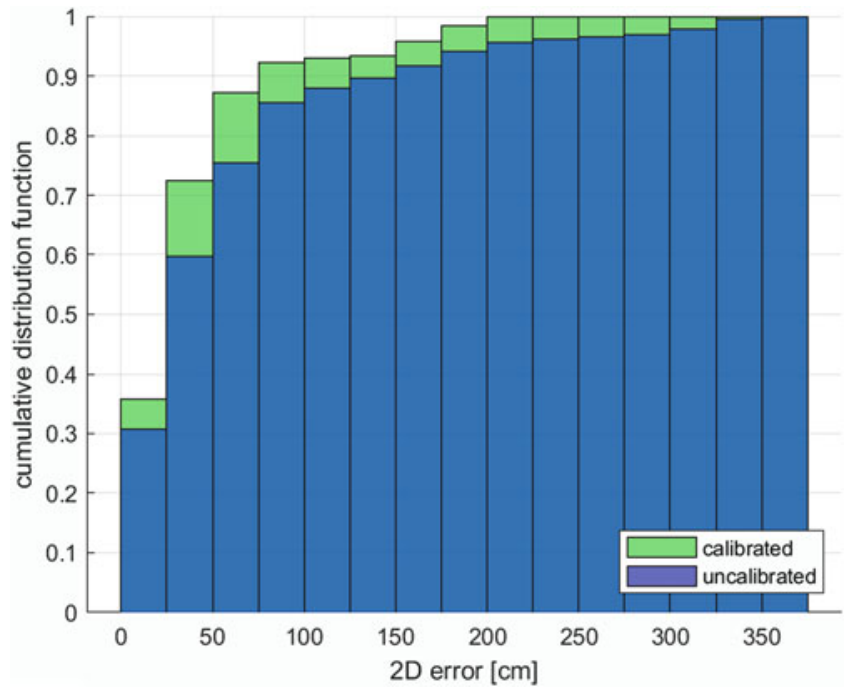

Fig. $3 \mathrm{CDF}$ of the $2 \mathrm{D}$ errors of the position estimates of the 35 checkpoints

that purpose the test area in the building was segmented in cells including rooms and sections of $4 \mathrm{~m}$ in length in the corridor as well as entrances or exits. The localization method chosen was location fingerprinting. In the training phase of the fingerprinting, 200 RSSI scans of the visible Access Points (APs) at different locations were simultaneously collected by 10 different mobile devices to be able to locate a user in the positioning phase who has scanned again for the APs. In this phase, Bayesian inference is applied to calculate the probability that a user is at a certain location given a specified observation. Then the most likely location of the mobile device can be estimated. Thereby the accuracy of the statistical distribution model directly affects the final performance of the probabilistic fingerprint positioning (see e.g. Xia et al. 2017). The authors in Li et al. (2018) proposed a statistical approach to localize the mobile user to room level accuracy based on the Multivariate Gaussian Mixture Model 

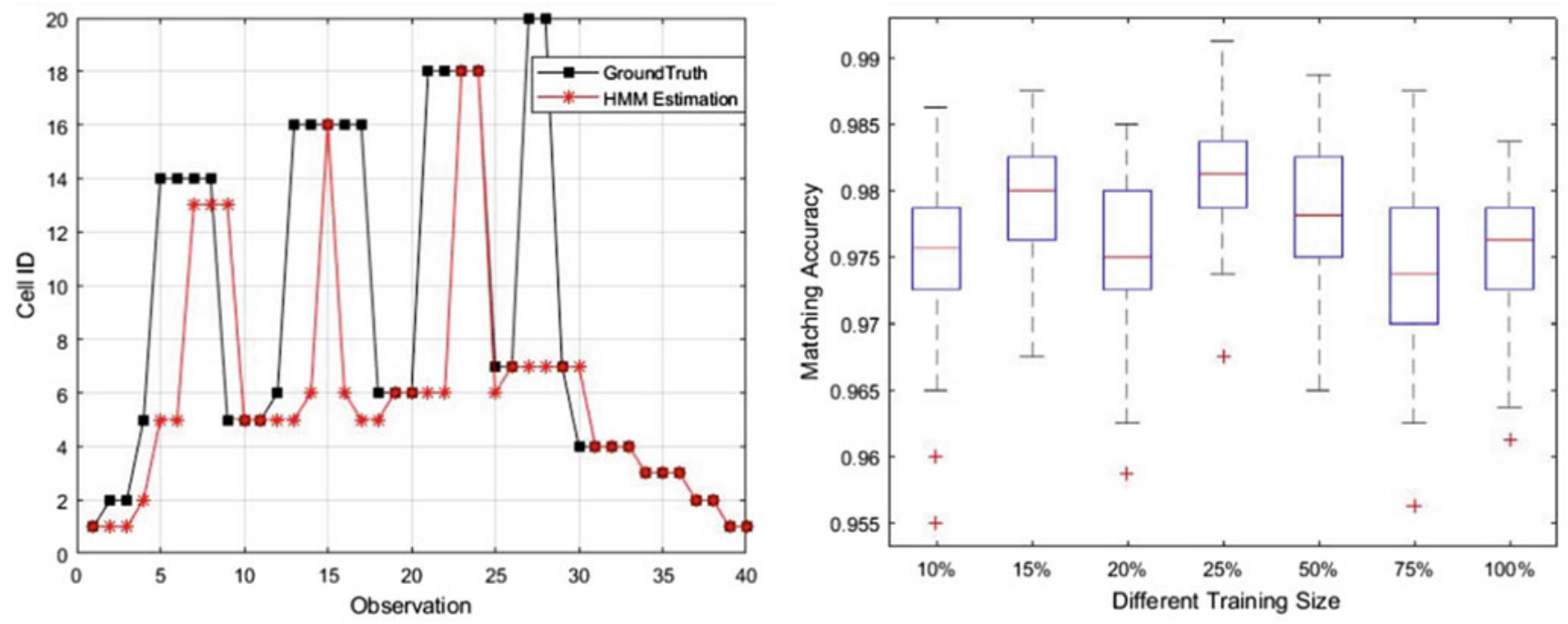

Fig. 4 Example of a kinematic walking trajectory (left) and matching probability rates for different training data sizes (right)

(MVGMM). A Hidden Markov Model (HMM) is applied to track the mobile user, where the hidden states comprise the possible room locations and the RSSI measurements are taken as observations. Due to the segmentation of the test area in different cells the transition matrix in the HMM is defined in such a way that only adjacent cells have nonzero transition probability while the transition probability between isolated cells is zero. In total, 11 kinematic walking trajectories were carried out with the different smartphones. Figure 4 (left) shows an example of an obtained trajectory of one smartphone user who moved in the study area between different defined cells. Figure 4 (right) presents the corresponding matching probabilities with different training sizes. The trajectories along the reference points could be obtained with matching success rates of up to $97 \%$. It can be seen that the proposed method is nearly insensitive to the size of the training samples, even presenting more robust localization accuracy to lower sample sizes. This result is similar to the work in Zhou (2006) where the authors found that, given dense training samples for the area may introduce more noise to distinguish from other areas. It can be finally summarized that the proposed system and algorithm demonstrated a reliable room location awareness system in a real public environment.

\section{$4 \quad$ Concluding Remarks and Outlook}

In the presented benchmarking measurement campaign, the main focus was on CL of different sensor platforms, i.e., vehicles, bicyclists and pedestrians, in GNSSdenied/challenged in-/outdoor and transitional environments.
For this paper, pedestrian users wearing a specially designed sensor helmet navigated jointly in a neighborhood in an indoor positioning application. An overview of the field experimental schemes, set-ups, characteristics and sensor specifications along with the main results for the positioning are presented and further details may be found in Kealy et al. (2019). In these analyses trajectories of pedestrians walking around in an indoor office environment could be obtained on the decimeter-level using UWB and with WiFi fingerprinting matching success rates of around $97 \%$ were achieved for assigning the user to the correct cell, i.e., either a room or section of the hallway. Further data processing and analyses of a CL solution is currently in progress and the results for UWB navigation presented in Gabela et al. (2019) indicate significant performance improvements of users navigating within a neighborhood. Positioning accuracies on the decimetre level are achieved for two moving users even at the end of the hallway where the geometry of the range measurement to the anchors is not the best. Ranges between the users constrain and improve the solution in this respect. Future work is especially concentrated on analyses of the localization accuracies and performance in the transitional and indoor environments. Apart from absolute localization of the users, dead reckoning with the inertial sensors is a further key element of future investigations. Especially the use of the smartphone sensors in combination with Wi-Fi and cameras is considered as a smartphone was mounted on the helmet which recorded at the same time Wi-Fi RSSI's, videos and the measurements of the inertial sensors as well as magnetometer and barometer. The extensive data set of the campaign are available for researchers from the joint IAG and FIG working group 
on request. The successful work of this Working Group in the past period will continue in the next years as a joint effort of IAG Sub-Commission 4.1 and FIG Working Group 5.5 .

\section{References}

Gabela J, Retscher G, Goel S, Perakis H, Masiero A, Toth CK, Gikas V, Kealy A, Koppanyi Z, Błaszczak-Bąk W, Li Y, Grejner-Brzezinska DA (2019) Experimental evaluation of a UWB-based cooperative positioning system for pedestrians in GNSS-denied environment. Sensors 19(23):5274. https://doi.org/10.3390/s19235274

GNSS Market Report (2017). https://www.gsa.europa.eu/system/files/ reports/gnss_mr_2017.pdf. Accessed Aug 2019

Kealy A, Retscher G, Gabela J, Li Y, Goel S, Toth CK, Masiero A, Błaszczak-Bąk W, Gikas V, Perakis H, Koppanyi Z, GrejnerBrzezinska DA (2019) A benchmarking measurement campaign in GNSS-denied/challenged indoor/outdoor and transitional environments, FIG Article of the Month, July. http://fig.net/resources/ monthly_articles/2019/kealy_etal_july_2019.asp

Li Y, Williams S, Moran B, Kealy A, Retscher G (2018) Highdimensional probabilistic fingerprinting in wireless sensor networks based on a multivariate Gaussian mixture model. Sensors 18(8):2602. https://doi.org/10.3390/S18082602
Masiero A, Fissore F, Guarnieri A, Pirotti F, Vettore A (2019) Aiding indoor photogrammetry with UWB sensors. Photogramm Eng Remote Sens 85(5):369-378. https://doi.org/10.14358/PERS.85.5. 369

Retscher G, Leb A (2019) Influence of the RSSI scan duration of smartphones in kinematic Wi-Fi fingerprinting. In: Proceedings of the FIG working week, 22-26 April, Hanoi, 15 pp

Retscher G, Kealy A, Gabela J, Li Y, Goel S, Toth CK, Masiero A, Błaszczak-Bąk W, Gikas V, Perakis H, Koppanyi Z, GrejnerBrzezinska DA (2020a) A benchmarking measurement campaign in GNSS-denied/challenged indoor/outdoor and transitional environments. J Appl Geod 14(2) (accepted). https://www.fig.net/fig2020/ technical_program.htm

Retscher G, Li Y, Kealy A, Gikas V (2020b) The need and challenges for ubiquitous Positioning, Navigation and Timing (PNT) using Wi-Fi. In: Proceedings of the FIG working week, 10-14 May, Amsterdam, $18 \mathrm{pp}$. https://fig.net/resources/proceedings/fig_proceedings/ fig2020/papers/ts05g/TS05G_retscher_li_et_al_10335.pdf

Xia S, Liu Y, Yuan G, Zhu M, Wang Z (2017) Indoor fingerprint positioning based on Wi-Fi: an overview. ISPRS Int J Geo-Information 6:135. https://doi.org/10.3390/ijgi6050135

Zhou R (2006) Wireless Indoor Tracking System (WITS). Aktuelle Trends in der Software Forschung, doIT Software-Forschungstag, dpunkt Verlag, Heidelberg, pp 163-177

Open Access This chapter is licensed under the terms of the Creative Commons Attribution 4.0 International Licence (http://creativecommons.org/ licenses/by/4.0/), which permits use, sharing, adaptation, distribution and reproduction in any medium or format, as long as you give appropriate credit to the original author(s) and the source, provide a link to the Creative Commons licence and indicate if changes were made.

The images or other third party material in this chapter are included in the chapter's Creative Commons licence, unless indicated otherwise in a credit line to the material. If material is not included in the chapter's Creative Commons licence and your intended use is not permitted by statutory regulation or exceeds the permitted use, you will need to obtain permission directly from the copyright holder. 